\title{
Piloleiomyosarcoma in cats: Histological and immunohistochemical features
}

Veterinary Pathology

I-6

(C) The Author(s) 2021

Article reuse guidelines:

sagepub.com/journals-permissions DOI: I0.1 |77/030098582 I I042582

journals.sagepub.com/home/vet

(9)

\author{
Francisco Rodríguez Guisado' $\stackrel{\odot}{\infty}$ and Pedro Luis Castro'
}

\begin{abstract}
This study describes the histomorphology and immunohistochemical profile of 9 cases of feline piloleiomyosarcoma. Cats ranged in age from 7 to 16 years (mean 10), and tumors were 7 to $24 \mathrm{~mm}$ in diameter (mean 15). Tumors were composed of fusiform cells that were haphazardly arranged or in variably sized interwoven bundles. Neoplastic cells had eosinophilic and fibrillar cytoplasm, and elongated blunt-ended nuclei. Entrapment of hair follicles and absence of vascular components support an origin from the smooth muscle cells of the arrector pili. Additional findings included bizarre nuclei and giant cells (7/9 cases), atypical mitoses ( $7 / 9$ cases), ulceration ( $3 / 9$ cases), and intratumoral necrosis (6/9 cases). Neoplastic cells expressed calponin, desmin, $\alpha$ smooth muscle actin, and vimentin, but not CDI8, CD3I, cytokeratins, glial fibrillary acidic protein, neuron-specific enolase, Melan A, p63, or S-100 protein. Surgical excision was curative in 6/9 cases, with local recurrence in 2/9 cases and metastasis to local lymph nodes in $1 / 9$ case. Clinical outcome was influenced by mitotic count, infiltration of subcutaneous tissue, and intensity of nuclear immunolabeling for $\mathrm{p} 53$.
\end{abstract}

\section{Keywords}

cat, immunohistology, integument, piloleiomyosarcoma, skin, smooth muscle, spindle cell, surgical pathology

Malignant smooth muscle tumors of the skin are infrequently reported in humans and animals. ${ }^{1-4,6,15}$ They may arise from arrector pili muscles, from blood vessel walls, or from specialized muscles in genital areas. ${ }^{4,6,7,12}$ Differentiation from other connective tissue tumors may be difficult in some cases on the basis of light microscopy alone, and immunohistochemistry is often necessary to confirm the diagnosis. ${ }^{1,7}$ Dermal smooth muscle tumors have been sporadically described in the dog, cat, ferret, and horse. ${ }^{1,4,7,14}$ In cats, with the exception of 1 piloleiomyosarcoma and 4 angioleiomyosarcomas, ${ }^{8,12}$ the features of cutaneous leiomyosarcomas have not been documented.

Histologically, these tumors are composed of interlacing bundles of neoplastic smooth muscle cells. Criteria of malignancy in humans include mitotic rate, cellular density, nuclear atypia, and intratumoral necrosis. Leiomyosarcomas in humans include myxoid, epithelioid, and pleomorphic subtypes, the latter being the only recognized pattern in the veterinary literature, ${ }^{1,4,7,12,14}$ some of which resemble anaplastic sarcomas. Leiomyosarcomas in cats may be underdiagnosed as they can mimic other more common spindle cell tumors of the feline skin, ${ }^{4,7,8,12,15}$ especially fibrosarcomas and nerve sheath tumors.

This study characterizes the histological, immunohistochemical, and clinical features of feline piloleiomyosarcoma, comparing them with its benign counterpart.

\section{Materials and Methods}

The study population was 9 cats from which biopsies were submitted between October 2014 and December 2020 to the Veterinary School of Las Palmas de Gran Canaria, Spain. This number represented $0.3 \%(9 / 2819)$ of all feline cutaneous tumors in the same period. Cutaneous neoplasms with a morphological description including the terms "spindle cell tumor," "smooth muscle tumor," or "unclassified sarcoma" were selected from the database, and the diagnosis was confirmed by histological review of hematoxylin and eosinstained sections. Inclusion criteria included a morphological diagnosis of malignant cutaneous spindle cell tumor with immunohistochemical profile of smooth muscle origin, determined by cytoplasmic staining for the myogenic components desmin and $\alpha$-smooth muscle actin. Based on the microscopic features suggestive of malignancy previously reported in

\footnotetext{
'University of Las Palmas de Gran Canaria, Arucas, Gran Canaria, Spain

Supplemental material for this article is available online.

Corresponding Author:

Francisco Rodríguez Guisado, Department of Morphology, Veterinary School, University of Las Palmas de Gran Canaria, Trasmontaña, Arucas 354I3, Gran Canaria, Spain.

Email: francisco.guisado@ulpgc.es
} 
Table I. Clinical and histological data of feline piloleiomyosarcoma (cases I-9) and piloleiomyoma (cases I0-14).

\begin{tabular}{|c|c|c|c|c|c|c|c|c|}
\hline Case & Breed $^{a}$ & Age (years) & Sex & Location & Mitoses $^{c}$ & Tumor size $(\mathrm{mm})$ & Additional findings ${ }^{d}$ & Clinical outcome \\
\hline 2 & $\mathrm{DSH}$ & 8 & SF & Forelimb & 5.9 & 12 & $\mathrm{~F} / \mathrm{N} / \mathrm{U}$ & No recurrence \\
\hline 4 & DSH & 11 & SF & Shoulder & 6.5 & 11 & $\mathrm{~N} / \mathrm{U}$ & No recurrence \\
\hline 5 & DSH & 9 & CM & Axilla & 18.4 & 21 & $\mathrm{~A} / \mathrm{F} / \mathrm{N} / \mathrm{S} / \mathrm{U}$ & Lymph node metastasis, 5 months \\
\hline 6 & DSH & 7 & $\mathrm{~F}$ & Trunk & 16.7 & 24 & $\mathrm{~A} / \mathrm{F} / \mathrm{N} / \mathrm{S}$ & Recurred, 3 months \\
\hline 9 & DSH & 9 & $\mathrm{~F}$ & Hind limb & 5.7 & 18 & $\mathrm{~A} / \mathrm{N}$ & No recurrence \\
\hline 10 & DSH & 8 & $\mathrm{~F}$ & Trunk & 0.3 & II & - & No recurrence \\
\hline II & DSH & 9 & $\mathrm{~F}$ & Shoulder & 0.7 & 9 & - & No recurrence \\
\hline 12 & DLH & 7 & $M$ & Hind limb & 0.3 & 14 & - & No recurrence \\
\hline 13 & DSH & 10 & $\mathrm{~F}$ & Trunk & 1.7 & 8 & - & No recurrence \\
\hline 14 & DSH & 12 & SF & Trunk & I & 13 & - & No recurrence \\
\hline
\end{tabular}

${ }^{a} \mathrm{DSH}$, domestic short haired; DLH, domestic long haired.

${ }^{\mathrm{b}} \mathrm{F}$, female; SF, spayed female; $\mathrm{M}$, male; $\mathrm{CM}$, castrated male.

'Mitotic figures $/ 2.37 \mathrm{~mm}^{2}$.

${ }^{\mathrm{d}} \mathrm{A}$, abnormal mitosis; $\mathrm{F}$, inflammatory infiltration; $\mathrm{N}$, intratumoral necrosis; $\mathrm{S}$, invasion of subcutaneous tissue; $\mathrm{U}$, ulceration.

humans ${ }^{5}$ and domestic animals, ${ }^{4,12,14}$ histological criteria used in the current study to separate piloleiomyosarcomas from piloleiomyomas included the presence of $>2$ mitoses $/ 2.37 \mathrm{~mm}^{2}$, and at least 3 out of the following 4 findings: (1) invasion of the dermis and/or subcutaneous tissue, (2) intratumoral necrosis, (3) high cellularity, and (4) nuclear pleomorphism and bizarre mitoses. In order to compare the histological and immunohistochemical findings, 5 feline piloleiomyomas were included in the study. Twenty-two suspected cases, including histiocytic sarcoma, fibrosarcoma, and nerve sheath tumor, were excluded on the basis of morphological and immunohistochemical evaluations.

Tumor were submitted fixed in $10 \%$ buffered formalin and were then trimmed, embedded in paraffin wax, sectioned at 4 $\mu \mathrm{m}$, and stained with hematoxylin and eosin and Masson's trichrome. Paraffin blocks were available for immunohistochemical analysis. Mitotic figures were counted in 30 highpower fields (HPF; diameter of the field of view $=0.55 \mathrm{~mm}$; $40 \times$ objective and $10 \times$ ocular FN $22 \mathrm{~mm}$; Olympus BX40 microscope), and the results expressed as the mean of mitotic figures/10 high power fields $\left(2.37 \mathrm{~mm}^{2}\right)$.

Immunohistochemistry (Suppl. Table S1) was performed manually on deparaffinized sections using the avidin-biotinperoxidase complex technique for calponin (clone CALP, product MABT1504; Dako), cytokeratins (clone AE1/AE3, product MAB3412; Dako), desmin (clone D33, product 243M-1; Dako), glial fibrillary acidic protein (GFAP; polyclonal, product AB5804; Dako), Melan A (clone A103, product 281M-8; Dako), neuron-specific enolase (NSE; clone BBS/ NC/VI-H14, product IHCR2014-6; Dako), $\alpha$-smooth muscle actin ( $\alpha$-SMA; clone 1A4, product A5228; Dako), S-100 protein (polyclonal, product S2644; Dako), vimentin (clone V9, product V6389; Dako), CD31 (clone JC70, product 131M-9; Dako), CD18 (clone CA1.4E9, product MCA1780; Bio-Rad), p53 protein (clone PAB240, product AB26; Abcam), and epithelial/myoepithelial p63 marker (clone 4E4, product AB735; Abcam). Manufacturer's datasheets and/or internal controls for all antibodies used in the study demonstrated cross-reactivity with feline tissues. A biotinylated rabbit antimouse or swine anti-rabbit IgG (Vector Laboratories), diluted 1:200, was applied as a secondary reagent. An ABC complex (Vector) diluted 1:50 was applied as the third reagent. Sections were incubated for 5 minutes with 3,3'-diaminobenzidine tetrahydrocloride (Sigma) $0.035 \%$ in TBS containing $\mathrm{H}_{2} \mathrm{O}_{2} \quad 0.1 \%$. After rinsing in tap water, slides were lightly counterstained with Harris's hematoxylin and mounted under DPX mountant (BDH Laboratory Supplies) for microscopy.

Feline cutaneous tumors with fusiform cells that can resemble the morphology of leiomyosarcoma, such as amelanotic melanoma, hemangiosarcoma, fibrosarcoma, nerve sheath tumor, and vaccine-associated sarcoma, were also run using the same immunohistochemical procedures. Appropriate positive control of leiomyosarcomas from the reproductive tract, and negative control of substitution of primary antibody by phosphate-buffered saline, were done in parallel with each staining run, and internal controls were also present. The percentages of immunoreactive cells for each antibody and tumor were calculated by manually counting 1000 cells in randomly selected areas. For cytoplasmic markers, due to the difficulty in determining the cellular borders, positivity was identified as reactivity surrounding the nuclei. Because of the low number of cases, no statistical analysis was performed.

Clinical information was obtained from accompanying biopsy submission forms, and follow-up data were retrieved from the clinical files and/or obtained by phone calls to the referring veterinarians or owners. Recurrence and metastasis were determined by clinical examination of the primary site of surgery and regional lymph nodes, including aspiration cytology and radiographic evaluation. Age, breed, sex, and clinical outcome are summarized in Table 1. 


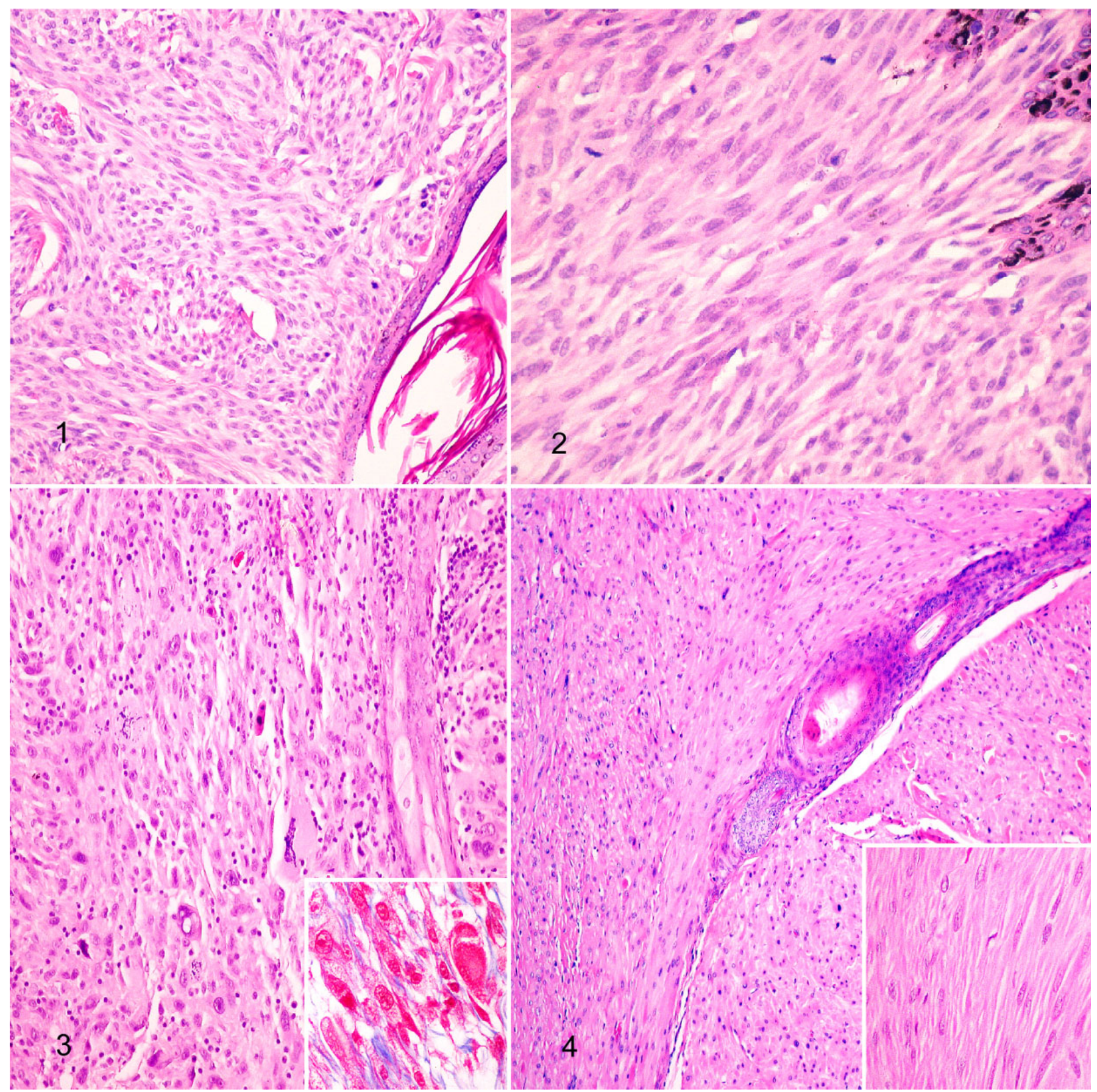

Figures I-3. Piloleiomyosarcoma, skin, cat. Hematoxylin and eosin (HE). Figure I. Case 3. Dermal growth of neoplastic cells abutting a hair follicle. HE. Figure 2. Case 4. Interlacing bundles of elongated cells with blunt-ended nuclei and frequent mitoses. HE. Figure 3. Case 6. Pleomorphic pattern, with round to spindle cells, giant cells, and atypical mitoses admixed with lymphocytes and plasma cells. HE. Inset: neoplastic cells stain red with Masson's trichrome. Figure 4. Piloleiomyoma, skin, cat. Spindle cells are arranged in uniform bundles with low cellularity, atypia, and mitotic activity. Case II. Inset: higher magnification. HE.

\section{Results}

The cats had a history of cutaneous growths that developed in a period of 1 to 3 weeks. Most tumors were located on the limbs ( 5 cases); the others arose on the shoulder, axilla, trunk, and neck (one case in each site). Tumors were solid, circumscribed, or locally invasive, and white to gray, with a homogeneous or multilobular appearance when sectioned.

Histologically (Figs. 1-3), lesions were located within the dermis and compressed or entrapped the adjacent cutaneous adnexa, either leaving an area of dermal collagen or directly 
contacting epithelial cells. Intratumoral foci of necrosis, with variable extension, was present in 6/9 tumors, and the overlying epidermis was ulcerated in 3/9 cases. Invasion of the subcutaneous tissue was observed in $3 / 9$ cases, which was related to clinical outcome, demonstrated by recurrence ( $2 / 3$ cases) and lymph node metastases ( $1 / 3$ case). Various numbers of inflammatory cells, consisting of neutrophils, lymphocytes, and plasma cells, infiltrated 7/9 tumors.

Neoplastic cells formed interlacing fascicles supported by a small amount of fibrovascular stroma. There were no findings indicating an origin of neoplastic cells from the media of the dermal vasculature or from intratumoral proliferation of vessels. Tumor cells had indistinct cell borders. The cytoplasm was abundant, lightly acidophilic, and had fine longitudinally arranged fibrils, and perinuclear halos in cross sections. Longitudinal, red-purple fibrils in the cytoplasm were visualized in Masson's trichrome stained sections, and fibroblasts and collagen intermingled with the smooth muscle bundles. Nuclei were pleomorphic, elongated or round, with blunt ends in longitudinal sections, coarse or granular chromatin, and prominent basophilic nucleoli. Bizarre nuclei and scattered multinucleated neoplastic cells were observed in 7/9 cases.

Piloleiomyomas consisted of well-circumscribed growths composed of smooth muscle cells arranged in regular bundles, without apparent atypia, multinucleation, or intratumoral necrosis, and with occasional mitotic figures $\left(0-2 / 2.37 \mathrm{~mm}^{2}\right.$, mean 0.8; Fig. 4). Unlike its malignant counterpart, most tumors were located on the trunk ( 3 cases), followed by the shoulder and limbs (one case in each site).

Cats with piloleiomyosarcomas ranged from 7 to 16 years (mean 10.5), and tumors were 7 to $24 \mathrm{~mm}$ in diameter (mean 14.9). Follow-up outcomes did not show differences in relation to breed, age, and sex. After surgical excision, no recurrence or metastases were recorded in $6 / 9$ cases. In contrast, the 3 tumors with $>12$ mitoses $/ 2.37 \mathrm{~mm}^{2}$ had either recurrence ( $2 / 9$ cases) or developed regional lymph node metastasis (1/9 case) confirmed by aspiration cytology and immunohistochemistry. Piloleiomyomas affected slightly younger cats (6-12 years, mean 8.4) and were smaller (8-13 mm, mean 11); surgical excision was curative with a follow-up period of 14 to 35 months.

Immunohistochemical results (Suppl. Table S2 and Figs. 5-8) showed that $>55 \%$ neoplastic cells in all tumors included in the study had cytoplasmic expression of calponin, desmin, $\alpha$-SMA, and vimentin, and showed nuclear positivity for $\mathrm{p} 53$ protein. Neoplastic cells did not express the other cellular markers evaluated, which included CD18, CD31, cytokeratins, GFAP, NSE, Melan A, p63, and S-100 protein. Piloleiomyosarcomas that had immunolabeling of $>65 \%$ of neoplastic cells for p 53 recurred or developed regional lymph node metastasis.

\section{Discussion}

Dermal tumors arising from smooth muscle are uncommon in dogs and cats. ${ }^{4,6,12,15}$ The criteria for malignancy in human smooth muscle tumors include high cellular density, pleomorphism, giant cells, and $>2$ mitoses $/ 2.37 \mathrm{~mm}^{2} .1,5,14,17$ In the current study, mitotic count varied from 4 to 18 , and there was correlation with the clinical outcome. Cases with $>12$ mitoses $/ 2.37 \mathrm{~mm}^{2}$ recurred or metastasized within a follow-up period of 3 to 8 months.

There are few differential diagnoses for well-differentiated leiomyosarcomas. ${ }^{4,7,12}$ Thus, the fascicular growth pattern of large neoplastic cells with fibrillar acidophilic cytoplasm, and elongated nuclei with blunt poles, are characteristic features. Although it is difficult to unequivocally separate benign from malignant smooth muscle tumors, cellularity, cytoplasmic and nuclear pleomorphism, multinucleation, mitotic counts, intratumoral necrosis, and evidence of invasion are features that support malignancy. ${ }^{4,6,12}$ In addition, it may be challenging to distinguish poorly differentiated leiomyosarcomas from other more common feline spindle-cell sarcomas. ${ }^{4,7,14}$ Histomorphological features of the tumors in the present study are consistent with those of piloleiomyosarcoma. The entrapment of hair follicles and the absence of vascular components, including lack of CD31 immunoreaction, support an origin from the smooth muscle cells of the arrector pili. ${ }^{7,12}$ These tumors were not genital leiomyosarcomas based on their locations. In humans, piloleiomyosarcomas are usually encountered on the limbs showing, when located in the dermis, recurrence rates of $30 \%$ to $50 \%$, and uncommonly metastasize, with higher frequency of recurrence and metastases for tumor with subcutaneous involvement. ${ }^{9,13,17}$ In the current study, 3 tumors (cases 1, 5, and 6) extended into the subcutaneous tissue, which was associated with a worse clinical outcome.

Desmin and $\alpha$-SMA were expressed by neoplastic cells in this series, and are characteristic markers of myogenic differentiation, making them useful markers of smooth muscle tumors in human and veterinary pathology. ${ }^{1,3,7,12}$ In spite of the fact that a number of authors have suggested $\alpha$-SMA is a more reliable marker than desmin in humans and dogs, ${ }^{2,10,12}$ and that some poorly differentiated smooth muscle tumors have failed to stain with these markers, ${ }^{2,4}$ the similar intensity in the expression of desmin and $\alpha$-SMA in our cases demonstrate the usefulness of including both antibodies for the diagnosis of piloleiomyosarcomas in cats. Differences in the affinity of primary antibodies and tissue immunoreactivity due to long-term fixation and/or antigen retrieval methods have been reported to influence immunohistochemical characterization of smooth muscle tumors. ${ }^{2,4}$

Calponin is a cytoskeleton-associated actin-binding protein that may be a more specific myogenic marker than either desmin or $\alpha$-SMA. ${ }^{4}$ Piloleiomyosarcomas in this study expressed calponin at lower intensity than desmin and $\alpha$-SMA, which is consistent with the observations in human tumors. ${ }^{4,13}$ Although calponin also immunoreacts with myoepithelial cells, ${ }^{4,7}$ the absence of immunolabeling for p63 supports the contention that tumors in this series were not of myoepithelial origin. Finally, the immunoreaction with vimentin, a widely distributed intermediate filament with strong positivity in cutaneous leiomyosarcomas of $\operatorname{dog} s^{1,4}$ and ferrets, ${ }^{14}$ supports the mesenchymal origin. ${ }^{7,12}$ While vascular walls and arrector pili 


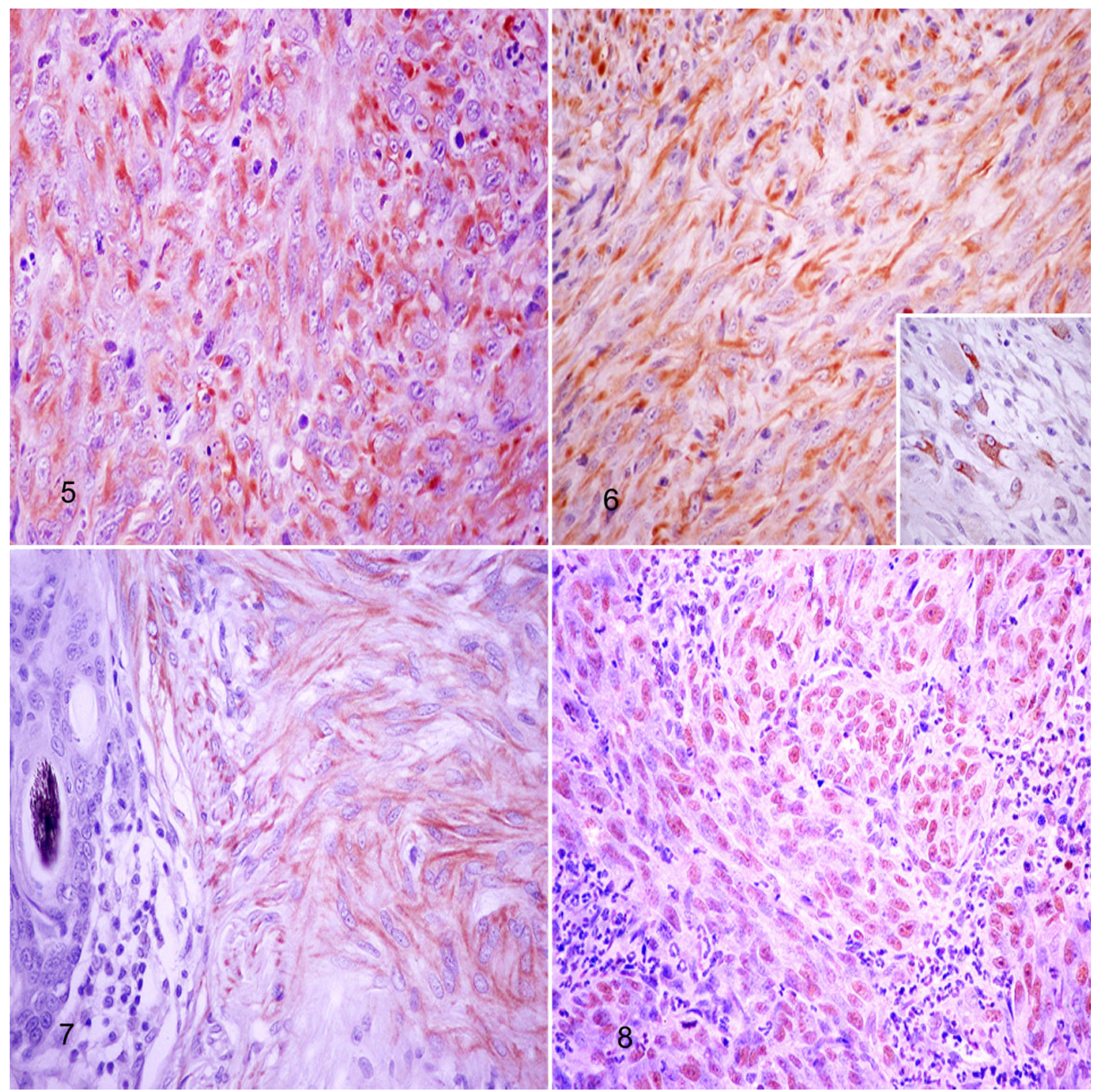

Figures 5-8. Piloleiomyosarcoma, skin, cat. The neoplastic cells have cytoplasmic immunolabeling for vimentin (Fig. 5, case 5), desmin in elongated and giant cells (Fig. 6, case 8; inset: higher magnification), calponin (Fig. 7, case 9), and nuclear immunostaining for p53 protein (Fig. 8, case I). Immunohistochemistry, avidin-biotin-peroxidase complex method.

muscles provide an appropriate internal control for desmin and $\alpha$-SMA, vimentin immunoreaction may also serve to evaluate the quality of tissue immunoreactivity. ${ }^{4}$

Expression of mutant p53 and its association with oncogenesis have been reported in canine and feline tumors. ${ }^{4,7,16}$ Mutations of p53 tumor suppressor gene, which is critical in inducing apoptosis, have been associated with leiomyosarcomas in humans. ${ }^{9,11}$ P53 mutations can be demonstrated by immunohistochemistry due to the overexpression of abnormal gene product. The difficulties in clearly differentiating leiomyoma from leiomyosarcoma, which may in some cases rely upon subjective criteria, prompted the investigation of the immunohistochemical expression of this marker. In humans, strong staining of p53 was associated with malignant transformation and aggressive behavior of cutaneous leiomyosarcomas. ${ }^{11}$ The worse outcome in cats with $>65 \%$ p53-positive cancer cells compared 
to $<5 \%$ positivity in the piloleiomyomas included in this study, support the use of p53 as a prognostic indicator.

Negative labeling with S-100, NSE, GFAP, Melan A, and CD31 allowed differentiation of piloleiomyosarcomas from other spindle cell neoplasms frequently seen in cats, such as nerve sheath tumors, spindle cell amelanotic melanoma, and hemangiosarcoma. ${ }^{4,12}$ Finally, the leukocyte marker CD18 served to exclude histiocytic neoplasms that can mimic pleomorphic leiomyosarcoma.

In conclusion, this study with a limited number of cases shows that piloleiomyosarcoma occurs in adult cats, without apparent differences in behavior related to breed, age, or sex. In comparison with piloleiomyoma, piloleiomyosarcoma appeared in older cats, was larger, and was mainly located on the limbs instead of in the trunk. Immunohistological analysis may be required to accurately differentiate this neoplasm from other feline spindle cell malignancies. Clinical outcome was influenced by mitotic count, invasion of subcutaneous tissue, and intensity of nuclear immunolabeling for $\mathrm{p} 53$.

\section{Declaration of Conflicting Interests}

The author(s) declared no potential conflicts of interest with respect to the research, authorship, and/or publication of this article.

\section{Funding}

The author(s) received no financial support for the research, authorship, and/or publication of this article.

\section{ORCID iD}

Francisco Rodríguez Guisado (D) https://orcid.org/0000-0002-4968-5333

\section{References}

1. Aihara N, Sugiyama J, Babah, et al. Multiple cutaneous pleomorphic leiomyosarcoma in a dog. J Vet Med Sci. 2019;81(11):1564-1566.
2. Andreasen CB, Mahaffey EA. Immunohistochemical demonstration of desmin in canine smooth muscle tumors. Vet Pathol. 1987;24(3):211-215.

3. Bellezza G, Sidoni A, Cavaliere A, et al. Primary cutaneous leiomyosarcoma: a clinicopathological and immunohistochemical study of 7 cases. Int J Surg Pathol. 2004;12(1):39-44.

4. Cooper BJ, Valentine BA. Tumors of muscle. In: Meuten DJ, ed. Tumors in Domestic Animals. 5th ed. Wiley Blackwell; 2017:425-466.

5. Fields JP, Helwig EB. Leiomyosarcoma of the skin and subcutaneous tissue. Cancer. 1981;47(1):156-169.

6. Finnie JW, Leong AS, Milios J. Multiple piloleiomyomas in a cat. J Comp Pathol. 1995;113(2):201-204.

7. Gross TL, Ihrke PJ, Walder EJ, et al. Smooth muscle and skeletal muscle tumors. In: Skin Diseases of the Dog and Cat: Clinical and Histopathological Diagnosis. 2nd ed. Blackwell Science; 2005:778-785.

8. Jacobsen MC, Valentine BA. Dermal intravascular leiomyosarcoma in a cat. Vet Pathol. 2000;37(1):100-103.

9. Jensen ML, Jensen OM, Michalski W, et al. Intradermal and subcutaneous leiomyosarcoma: a clinicopathological and immunohistochemical study of 41 cases. J Cutan Pathol. 1996;23(5):458-463.

10. Kaddu S, Beham A, Cerroni L, et al. Cutaneous leiomyosarcoma. Am J Surg Pathol. 1997;21(9):979-987.

11. Konomoto T, Fukuda T, Hayashi K, et al. Leiomyosarcoma in soft tissue: examination of p53 status and cell proliferating factors in different locations. Human Pathol. 1998;29(1):74-81.

12. Liu SM, Mikaelian I. Cutaneous smooth muscle tumors in the dog and cat. Vet Pathol. 2003;40(6):685-692.

13. Massi D, Franchi A, Alos L, et al. Primary cutaneous leiomyosarcoma: clinicopathological analysis of 36 cases. Histopathology 2010;56(2):251-262.

14. Mikaelian I, Garner MM. Solitary dermal leiomyosarcomas in 12 ferrets. J Vet Diagn Invest. 2002;14(3):262-265.

15. Miller MA, Nelson SL, Turk JR, et al. Cutaneous neoplasia in 340 cats. Vet Pathol. 1991;28(5):389-395.

16. Nambiar PR, Jackson ML, Ellis JA, et al. Immunohistochemical detection of tumor suppressor gene $\mathrm{p} 53$ protein in feline injection site-associated sarcomas. Vet Pathol. 2001;38(2):236-238.

17. Raj S, Calonje E, Kraus M, et al. Cutaneous pilar leiomyoma: clinicopathologic analysis of 53 lesions in 45 patients. Am J Dermatopathol. 1997;19(1):2-9. 